\section{SISTEMAS AQUOSOS BIFÁSICOS UMA FERRAMENTA SUSTENTÁVEL PARA A EXTRAÇÃO DE ÁCIDO CLAVULÂNICO A PARTIR DE DIFERENTES FONTES}

\author{
Jorge F. B. Pereira ${ }^{1}$, Valéria C. Santos-Ebinuma ${ }^{1}$, José A. C. Teixeira ${ }^{2}$, Adalberto \\ Pessoa $\mathrm{Jr}^{3}$
}

${ }^{1}$ Departamento de Bioprocessos e Biotecnologia, Faculdade de Ciências Farmacêuticas, UNESP-Univ. Estadual Paulista, Araraquara, SP, Brasil.

${ }^{2}$ Centro de Engenharia Biológica, Universidade do Minho, Campus de Gualtar, Braga, Portugal.

${ }^{3}$ Departamento de Tecnologia Bioquímico-Farmacêutica. Universidade de São Paulo, São Paulo, SP, Brasil.

E-mail para contato: jfbpereira@fcfar.unesp.br

\section{RESUMO}

O ácido clavulânico (AC) é um inibidor de b-lactamases que tem vindo a ser largamente utilizado na área médica. Embora seja de extrema importância, o desenvolvimento de processos alternativos de produção e purificação é ainda insignificante, sendo fundamental o estudo de técnicas de extração mais biocompatíveis, como os Sistemas Aquosos Bifásicos (SABs). Assim, este trabalho objetivou o estudo de Sistemas Aquosos Bifásicos baseados em polímeros como uma ferramenta alternativa para a extração de AC. Foram testados dois SPAB compostos por Polietileno Glicol (PEG) com massa molecular (M) de $4000 \mathrm{~g} / \mathrm{mol}$ e Poliacrilato de Sódio de $8000 \mathrm{~g} / \mathrm{mol}$, nos quais foi alterado o eletrólito indutor da formação de fases, em particular, sulfato de sódio $\left(\mathrm{Na}_{2} \mathrm{SO}_{4}\right.$,) e cloreto de sódio (NaCl). Ademais, este trabalho visou também avaliar a eficiência de extração do AC, bem como compreender o efeito dos contaminantes no processo de migração. Para tal, foi avaliada a extração do AC a partir de três fontes distintas: solução pura (99,9\%); solução comercial (60\%); diretamente a partir do sobrenadante de um meio fermentando de Streptomyces clavuligerus. Os resultados obtidos demonstraram que independentemente da fonte inicial do AC, ambos os SABs poliméricos promoveram uma partição preferencial do AC para a fase rica em PEG, sendo o coeficiente de partição maior nos sistemas com $\mathrm{Na}_{2} \mathrm{SO}_{4}$ do que com NaCl. Após identificar a grande capacidade de partição de AC, o SAB com $\mathrm{PEG} / \mathrm{NaPA} / \mathrm{Na}_{2} \mathrm{SO}_{4}$ foi também utilizado para avaliar a partição de proteínas presente no meio fermentado, sendo também obtida uma preferencial partição destas para a fase rica em PEG. Assim, apesar da baixa capacidade de purificação de AC frente a proteínas contaminantes, os SABs estudados demonstraram que podem ser uma técnica alternativa sustentável e bastante econômica para uma etapa inicial de clarificação/concentração de bioprodutos a partir de caldos fermentados. 


\section{SIMPÓSIO NACIONAL DE BIOPROCESSOS \\ XI SIMPÓSIO DE HIDRÓLISE ENZIMÁTICA DE BIOMASSA}

01 a 04 de setembro de 2015

Fortaleza, Ceará, Brasil

\section{INTRODUÇÃO}

Os tradicionais métodos de extração de moléculas apresentam alguns inconvenientes que impedem uma maior utilização em processos biotecnológicos, como por exemplo, longo tempo do processo, baixa seletividade e eficiência de extração. Assim, nos últimos anos, vêm-se ampliando o emprego dos métodos de extração líquido-líquido em sistemas aquosos bifásicos (SABs), com a finalidade de extrair biomoléculas (Rangel-Yagui et al., 2003). Os SABs podem ser formados pela adição de polímeros hidrofílicos ou de um polímero e um sal apropriado, formando os chamados Sistemas Poliméricos de Duas Fases Aquosas (SPDFA), aqui designados por Sistemas Bifásicos Aquosos Poliméricos (SABP) (Rangel-Yagui et al., 2003). Particularmente, o SABP Polietileno glicolPoliacrilato de Sódio (PEG/NaPA) apenas forma duas fases em determinadas condições, devido à necessidade das moléculas de NaPA estarem totalmente dissociadas $(\mathrm{pH}>7)$ e com uma quantidade suficiente de sal no sistema que facilite a compartimentalização do NaPA em uma das fases (Johansson et al., 2008a). Em suma, este SAPB PEG/NaPA apresenta algumas vantagens, como por exemplo a sua baixa viscosidade, fases claras bem definidas e possibilidade de reciclagem dos compentens formadores de fases. Ademais, como principal vantagem este SAPB apresenta alta concentração de água (aproximadamente 65-90\%) favorecendo a estabilidade de moléculas biologicamente ativas durante o processo de separação. Tendo em conta, que a estabilidade de biomoléculas é um dos principais problemas durante o processo de purificação, este tipo de SABs tem vindo a ser utilizados e propostos como uma alternativa interessante para a purificação de diversos tipos de biomoléculas (Johansson et al., 2008a, Johansson et al., 2008b).

$\mathrm{O}$ ácido clavulânico (AC), por exemplo, é um inibidor de 6 -lactamases quimicamente instável com elevadas taxas de degradação em meios básicos $(\mathrm{pH}>7,5)$ e ácidos $(\mathrm{pH}<4,5)$ (Bersanetti et al., 2005), o que leva a rendimentos de recuperação considerados baixos durante os processos de purificação. Este fármaco na sua forma de sal potássico (clavulanato de potássio) pode ser encontrado em associação com antibióticos para reverter o mecanismo de resistência de uma variedade de bactérias (Watve et al., 2000). Adicionalmente, estudos recentes têm demonstrado novas aplicações para este fármaco, tais como, modulação dos efeitos sobre o sistema nervoso central e em neuroproteção in vivo contra doença de Parkinson (Kost et al, 2011) e por interromper ações in vivo da morfina (Schroeder et al., 2014). Todas estas vantagens conduziram ao crescente interesse pelas empresas farmacêuticas para obtenção de AC puro a partir do caldo fermentado. Num trabalho recentemente publicado pelo nosso grupo (Pereira et al., 2012) demonstramos a capacidade dos SAPB compostos por PEG e NaPA em separar e extrair esta biomolécula a partir de meio fermentado de Streptomyces clavuligerus. Nesse sentido, vimos aqui mostrar com maior detalhe alguns dos fenômenos que contribuem e afetam a partição do AC a partir de diversas fontes, bem como apresentar soluções que possam permitir afinar e melhorar a purificação desse fármaco a partir de caldos fermentados num simples passo de extração.

\section{MATERIAIS E MÉTODOS}

\subsection{Materiais}

O NaPA de massa molar $8000 \mathrm{~g} / \mathrm{mol}$ (NaPA-8000) e o PEG de massa molar $4000 \mathrm{~g} / \mathrm{mol}$ (PEG-4000) foram adquiridos da Sigma ${ }^{\mathrm{TM}}$ (St. Louis, MO) e Merck, respectivamente. O AC na forma de sal potássio foi obtido de duas fontes diferentes: puro da Sigma (St. Louis, MO) (99\% de pureza), e grau 


\section{SIMPÓSIO NACIONAL DE BIOPROCESSOS \\ XI SIMPÓSIO DE HIDRÓLISE ENZIMÁTICA DE BIOMASSA}

01 a 04 de setembro de 2015

Fortaleza, Ceará, Brasil

farmacêutico da Galena ( $54 \%$ de pureza). Todos os outros reagentes utilizados foram de grau analítico. As soluções foram preparadas em tampão Mcllvaine pH 6,5.

\subsection{Produção do ácido clavulânico}

O AC foi obtido por cultivo submerso de Streptomyces clavuligerus ATCC 27064 em meio descrito por Ortiz et al. (2007). O bioprocesso foi realizado em agitador orbital a $28^{\circ} \mathrm{C}$ e $250 \mathrm{rpm}$ por $120 \mathrm{~h}$. Após o final do cultivo, o meio fermentado foi filtrado e o sobrenadante contendo o $\mathrm{AC}$ congelado em freezer a $-70^{\circ} \mathrm{C}$ para ser usado nos estudos de partição.

\subsection{Partição de ácido clavulânico por Sistema Polimérico de Duas Fases Aquosas}

Os SABs PEG/NaPA foram preparados em tampão Mcllvaine pH 6.5 nas seguintes condições: (a) $20 \%(\mathrm{~m} / \mathrm{m})$ de PEG-4000, 20\% (m/m) NaPA-8000 e 1,05\% (m/m) de NaCl; (b) $10 \%(\mathrm{~m} / \mathrm{m})$ PEG-4000, $20 \%(\mathrm{~m} / \mathrm{m}) \mathrm{NaPA}-8000$ e $6 \%(\mathrm{~m} / \mathrm{m}) \mathrm{Na}_{2} \mathrm{SO}_{4}$. Os componentes do sistema foram adicionados em tubos de vidro de $15 \mathrm{~mL}$ (massa total de 10,0 g). Estes sistemas foram definidos de acordo com resultados prévios (Pereira et al., 2012). Foram avaliadas três fontes de AC: meio fermentado, comercial e puro. Todas os SABs foram misturados a $8 \mathrm{rpm}$ durante $20 \mathrm{~min}$ a temperatura ambiente em agitador orbital. Os tubos foram mantidos em banho termorregulado (New Ethics, SP, Brazil), a $25^{\circ} \mathrm{C}$. Após a separação das fases, as fases superior e inferior foram coletadas cuidadosamente com auxílio de pipeta de Pasteur e a concentração de AC determinada em ambas as fases. $O$ comportamento da partição do AC foi avaliado em termos de coeficiente de partição, $K_{A C}$ :

$$
K_{A C}=\frac{C_{A C_{-} s}}{C_{A C_{-} i}}
$$

em que $C$ é a concentração de $A C\left(m g . L^{-1}\right)$ e os subscritos $s, i$, referem-se as fases superior (rica em PEG) e fundo (rica em NaPA), respectivamente. Adicionalmente foi também quantificado o coeficiente de partição total de proteínas $\left(K_{P}\right)$ e foi determinado de acordo com a equação 2 :

$K_{P}=\frac{C P_{s}}{C P_{i}}$

em que $C P_{s}$ e $C P_{i}$ são a concentração de proteína total $\left(\mathrm{g} \cdot \mathrm{L}^{-1}\right)$ na fase superior e na fase inferior, respectivamente.

\subsection{Determinação da concentração de ácido clavulânico e proteínas}

A concentração de AC foi determinada pelo método espectrofotométrico proposto por Bird et al., 1982. A determinação de proteínas foi realizada através do método BCA (bicinchoninic acid).

\section{RESULTADOS E DISCUSSÃO}

\subsection{Partição de ácido clavulânico por SABs PEG/NaPA}

Recentemente, nosso grupo de pesquisa (Pereira et al. 2012) otimizou e avaliou a partição do AC a partir de diferentes fontes, bem como em diferentes sistemas, e condições, como temperatura, concentração, etc. De acordo, com os resultados obtidos neste trabalho objetivamos avaliar e compreender os fatores que controlam a migração do AC obtidos a partir de diferentes fontes (comercial, pura e do meio fermentado), e como é que os contaminantes podem afetar essa 


\section{SIMPÓSIO NACIONAL DE BIOPROCESSOS \\ XI SIMPÓSIO DE HIDRÓLISE ENZIMÁTICA DE BIOMASSA}

01 a 04 de setembro de 2015

Fortaleza, Ceará, Brasil

mesma partição. Para tal, na Figura 1 compilamos e comparados os valores de coeficiente de partição obtidos para os SABs PEG/NaPA compostos por: (a) $20 \%(\mathrm{~m} / \mathrm{m})$ de PEG-4000, 20\% (m/m) NaPA-8000 e 1,05\% (m/m) de NaCl; (b) 10\% (m/m) PEG-4000, 20\% (m/m) NaPA-8000 e 6\% (m/m) $\mathrm{Na}_{2} \mathrm{SO}_{4}$.

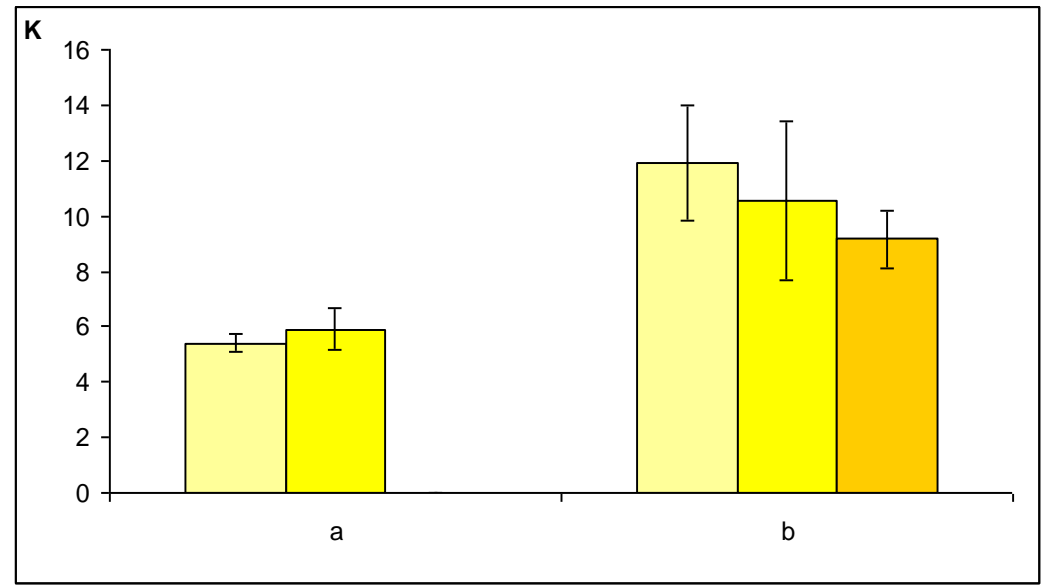

Figura 1. Coeficientes de partição do $\mathrm{AC}\left(K_{\mathrm{AC}}\right)$ obtido a partir de três fontes (comercial $\square$, pura $\square \mathrm{e}$ fermentado - ). Os SABs utilizados foram: (a) $20 \%(\mathrm{~m} / \mathrm{m})$ de PEG-4000, $20 \%(\mathrm{~m} / \mathrm{m})$ NaPA-8000 e $1,05 \%$ $(\mathrm{m} / \mathrm{m})$ de $\mathrm{NaCl}$; (b) $10 \%(\mathrm{~m} / \mathrm{m})$ PEG-4000, $20 \%(\mathrm{~m} / \mathrm{m}) \mathrm{NaPA}-8000$ e $6 \%(\mathrm{~m} / \mathrm{m}) \mathrm{Na}_{2} \mathrm{SO}_{4}$. As barras de erro correspondem ao intervalo de confiança de $95 \%$ nos valores obtidos.

Pela análise da Figura 1 é observado de imediato uma importante influência do tipo de eletrólito utilizado no $S A B s$ na partição do $A C$, independentemente da fonte de $A C$. Desse modo verificou-se que a partição do $A C$ foi sempre superior no sistema que utilizou $6 \%(\mathrm{~m} / \mathrm{m})$ de $\mathrm{Na}_{2} \mathrm{SO}_{4}$. Ademais, uma análise detalhada da partição do $A C$ relativamente à fonte da qual foi obtido o $A C$, permite verificar que os contaminantes que se encontram nas fontes originais podem afetar significativamente a partição do fármaco. Os resultados permitem compreender os mecanismos de partição de biomoléculas presentes em meios bastante complexos (p.ex. meios fermentados), ou produtos com alta taxa de contaminantes (como o fármaco comercial produzido pela Galena). Pela comparação da partição do AC comercial e puro, observa-se uma influência significativa dos contaminantes na partição, uma vez que em ambos os sistemas (a) e (b), a presença de $40 \%$ de contaminantes promoveu uma variação do $K_{\mathrm{Ac}}$, sendo observado um aumento da partição quando utilizado o $\mathrm{Na}_{2} \mathrm{SO}_{4}$, e uma pequena diminuição quando aplicado o sistema com $\mathrm{NaCl}$.

Por outro lado, quando avaliado a partição do AC proveniente de um bioprocesso, i.e. o AC obtido a partir do meio fermentado de Streptomyces clavuligerus, a influência dos contaminantes é ainda mais evidente (maior variação $K_{\mathrm{AC}}$ ). Pela Figura 1 é observado a diminuição da capacidade de extração do AC quando esse é recuperado do meio fermentado no SAB PEG/NaPA/ $/ \mathrm{Na}_{2} \mathrm{SO}_{4}$. Contudo, o resultado mais importante foi verificado no $S A B$ com o eletrólito mais fraco $(\mathrm{NaCl}), 0$ qual não foi efetivo na partição do $A C$, visto que formou uma larga interface $(6 \mathrm{~mL})$, originando uma fase superior pequena $(2 \mathrm{~mL})$ e uma fase inferior quase inexistente. Essa não definição de fases não permitiu avaliar qualquer parâmetro de extração do $A C$ a partir de meio fermentado.

De um modo geral, foi evidente que a utilização de SABs PEG/NaPA com uma concentração maior de eletrólitos com forte capacidade de solvatação, como é o caso do $\mathrm{Na}_{2} \mathrm{SO}_{4}$, permite uma 


\section{SIMPÓSIO NACIONAL DE BIOPROCESSOS \\ XI SIMPÓSIO DE HIDRÓLISE ENZIMÁTICA DE BIOMASSA}

01 a 04 de setembro de 2015

Fortaleza, Ceará, Brasil

manutenção da capacidade de extração do fármaco mesmo a partir de meios bastante complexos. No entanto, quando se utiliza uma concentração menor de sal $(2 \%, \mathrm{~m} / \mathrm{m})$, e sais mais fracos (com menor capacidade de salting-out), como o $\mathrm{NaCl}$, a eficiência do processo de biosseparação é muito desfavorecido, ocorrendo até a redução da capacidade de formação de duas fases imiscíveis. Anteriormente, Johansson et al. (2008b) demonstrou que para que ocorra a formação da região bifásica é necessário uma concentração mínima de sal. Neste caso, uma vez que nas misturas complexas, como meios fermentados, se encontram outros componentes (p.ex. proteínas), a quantidade de sal necessária para promover uma separação efetiva é possivelmente superior.

Apesar da diminuição do $K_{\mathrm{AC}}$ quando comparado aos sistemas puros e comerciais, os resultados do $\mathrm{SAB}$ com $\mathrm{Na}_{2} \mathrm{SO}_{4}$ evidenciaram uma grande capacidade para extrair o AC do meio fermentado, com um $K_{\mathrm{AC}} \approx 9,2$. Assim, considerando que no meio fermentado além do fármaco em estudo existem diversos metabólitos, em particular proteínas, foi também avaliada a partição das proteínas no sistema (b), PEG/NaPA/Na $\mathrm{SO}_{4}$. Os resultados são apresentados na Figura 2.

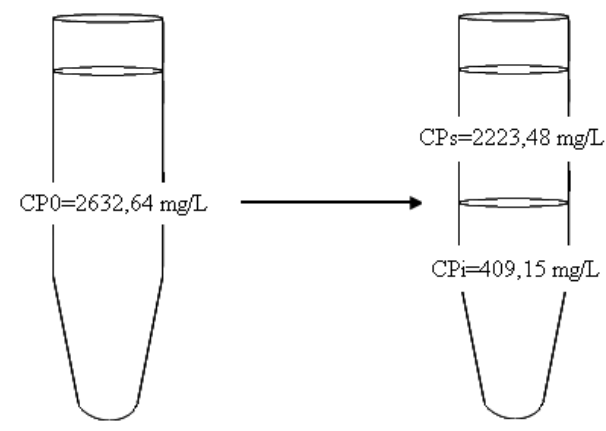

Figura 2. Diagrama representativo da concentração das proteínas totais no meio inicial $\left(\mathrm{CP}_{0}\right)$, na fase de superior $\left(\mathrm{CP}_{\mathrm{s}}\right)$ e na fase inferior $\left(\mathrm{CP}_{\mathrm{i}}\right)$.

A Figura 2 demonstra que, tal como para o AC, as proteínas migraram preferencialmente para a fase superior do sistema (rica em $P E G$ ), $K_{P} \approx 5,4$, não permitindo uma extração seletiva do fármaco relativamente aos contaminantes presentes no meio fermentado. No entanto, apesar da extração seletiva AC/proteínas não ter sido, importa realçar que outras moléculas contaminantes com maior interação pelo NaPA podem ter sido particionadas na fase inferior (clarificação de baixa resolução).

A partição das proteínas para a fase superior ou inferior depende do tipo de proteínas presentes no meio fermentado, do tipo e concentração de sais e polímeros. Assim, a otimização do processo de extração do AC no sistema PEG/NaPA passa pelo ajuste da concentração polimérica e de electrólito para afinar a seletividade AC/proteínas. Por outro lado, essa otimização depende também da caracterização do tipo de proteínas presentes na fase de PEG, bem como do desenvolvimento de etapas posteriores de re-extração. Os processos de re-extração têm sido muito estudados, e envolvem a utilização de outros tipos de SABs, p.ex. SABs compostos com Líquidos lônicos (LIs) e PEGs. Estes novos SABs com Lls têm se mostrado eficientes na extração seletiva de várias biomoléculas, mesmo em misturas de compostos similares como os alcalóides (Pereira et al., 2013), uma vez que com o desenho racional do LI utilizado pode afinar a migração do composto alvo para uma das fases em equilíbrio. 


\section{SIMPÓSIO NACIONAL DE BIOPROCESSOS \\ XI SIMPÓSIO DE HIDRÓLISE ENZIMÁTICA DE BIOMASSA}

01 a 04 de setembro de 2015

Fortaleza, Ceará, Brasil

\section{CONCLUSÃO}

A presença de contaminantes no meio fermentado afetou significativamente a partição do Ácido Clavulânico. O sistema composto por PEG-4000/NaPA-8000/ $/ \mathrm{Na}_{2} \mathrm{SO}_{4}$ apresentou uma grande capacidade para extrair o AC a partir do meio fermentado de Streptomyces clavuligerus, provando que este SAB pode ser utilizado como uma plataforma prévia para uma etapa de clarificação/concentração de fármacos produzidos por bioprocessos.

\section{REFERÊNCIAS}

Bersanetti P.A., Almeida R.M.R.G., Barboza M., Araujo M.L.G., Hokka C.O., 2005. Kinetic studies on clavulanic acid degradation. Biochem. Eng. J. 23, 31-36.

Bird A.E., Bellis J.M., Gasson B.C., 1982. Spectrophotometric Assay of Clavulanic Acid by Reaction with Imidazole. Analyst. 107, 1241-1245.

Pereira J.F.B., Santos V.C., Johansson H.O., Teixeira J.A.C., Pessoa Jr A., 2012. A stable liquid-liquid extraction system for a clavulanic acid using polymer-based aqueous two-phase system. Sep. Purif. Technol. 98, 441-450.

Pereira J.F.B., Santos V.C., Johansson H.O., Teixeira J.A.C., Pessoa Jr A., 2013. Aqueous biphasic systems composed of ionic liquids and polymers: A platform for the purification of biomolecules. Sep. Purif. Technol. 113, 83-89.

Johansson H.-O., Ishii M., M. Minagutti, Feitosa E., Penna, T.C.V., Pessoa-Jr, A., 2008a. Separation and partitioning of green fluorescent protein from Escherichia coli homogenate in poly(ethylene glycol)/sodium-poly(acrylate) aqueous two-phase systems. Sep. Purif. Technol. 62, 166-174.

Johansson H.-O., Magaldi F.M., Feitosa E., Pessoa-Jr, A., 2008b. Protein partitioning in poly(ethylene glycol)/sodium polyacrylate aqueous two-phase systems. J. Chromat. A 1178, 145-153.

Kost G.C., Selvara S., Lee Y.B., Kim D.J., Ahnn C., Singh B.B., 2011. Clavulanic acid increases dopamine release in neuronal cells through a mechanism involving enhanced vesicle trafficking. Neurosci. Lett. 504, 170-175.

Ortiz S.C.A., Hokka C.O., Badino A.C., 2007. Utilization of soybean derivatives on clavulanic acid production by Streptomyces clavuligerus. Enzyme Microb. Tech. 40, 1071-1077.

Rangel-Yagui CO, Lam H, Kamei DT,Wang DIC,Pessoa-Jr A, Blankschtein D., 2003. Glucose-6phosphate dehydrogenase partitioning in two-phase aqueous mixed (nonionic/cationic) micellar systems. Biotecnol. Bioeng. 82, 445-456.

Schroeder J.A., Tolman N.G., McKenna F.F., Watkins K.L., Passeri S.M., Hsua A.H., Shinna B.R., Rawls S.M., 2014. Clavulanic acid reduces rewarding, hyperthermic andlocomotor-sensitizing effects of morphine in rats:A new indication for an old drug? Drug Alcohol Depen. 142, 41-45.

Watve M.G., Shejval V., Sonawane C., Matapurkar M., Shouche Y., Patole M., Padnis N., Champhenkar A., Damle K., Karandikar S., Jog M., 2000. The "K" selected oligophilic bacteria: a key to uncultured diversity? Current Science 78, 1535- 1542. 